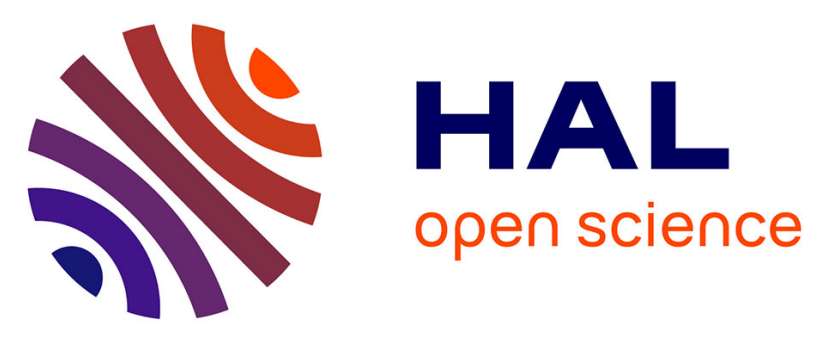

\title{
Maintenance Optimization of a Balanced System with Tolerance
}

Wenjin Zhu, Christophe Bérenguer

\section{To cite this version:}

Wenjin Zhu, Christophe Bérenguer. Maintenance Optimization of a Balanced System with Tolerance. ESREL 2021 - 31st European Safety and Reliability Conference, Sep 2021, Angers, France. pp.364-368, 10.3850/978-981-18-2016-8_534-cd. hal-03450091

\section{HAL Id: hal-03450091 https://hal.science/hal-03450091}

Submitted on 10 Dec 2021

HAL is a multi-disciplinary open access archive for the deposit and dissemination of scientific research documents, whether they are published or not. The documents may come from teaching and research institutions in France or abroad, or from public or private research centers.
L'archive ouverte pluridisciplinaire HAL, est destinée au dépôt et à la diffusion de documents scientifiques de niveau recherche, publiés ou non, émanant des établissements d'enseignement et de recherche français ou étrangers, des laboratoires publics ou privés.

\section{(1) (1) $\$$}

Distributed under a Creative Commons Attribution - NonCommercial - NoDerivatives $\mid 4.0$ 


\title{
Maintenance Optimization of a Balanced System with Tolerance
}

\author{
Wenjin Zhu \\ School of Mechanical Engineering, Northwestern Polytechnical University, China. E-mail: wenjin.zhu@nwpu.edu.cn \\ Christophe Bérenguer \\ Univ.Grenoble Alpes, CNRS, Grenoble-INP,GIPSA-lab, Grenoble, France. E-mail: christophe.berenguer@grenoble-inp.fr
}

In this paper a pair of two rail-wheels which is submitted to degradation due to the unbalanced vibration and wear during long time running is studied. The pair of wheels works under the minimum allowable value of flange thickness and tread diameter. The thickness of flange and the diameter of wheel tread are dependent on each other by maintenance action, referred as reprofiling in this study. In order to keep the thickness of flange above a given value for the sake of safety, when the thickness of flange decreased, the diameter of the tread will be reduced by reprofiling (rotary cut) so as to increase the thickness of flange.

The natural degradation of thickness of flange and the diameter are modelled by Wiener process. The dependence between two wheels is based on the wheel diameter difference. The inspection of the wheels is period and perfect. When the thickness of flange degrades under the given threshold, or when the diameter of anyone of the pair of the wheels degrades under the given threshold, the system is regarded as failed. When the difference of diameters between two rail-wheel pairs is greater than the tolerance or the thickness of the flange reduces over the preventive maintenance threshold, a preventive maintenance activity is planned. Hence the preventive maintenance threshold and the maintenance period are optimized by simulations.

Keywords: Wiener process, symmetric system, degradation, maintenance, tolerance, rail-wheel.

\section{Introduction}

Balanced systems are class of systems with special structure which is characterized by symmetrical structure or sometimes a structurally symmetrical function and performance (Gao et al. (2019), Zhao et al. (2020), Fang and Cui (2021)). Take a multi-engine fighter as an example: if one engine on one side is malfunctioning, then the engine on the other side at the symmetrical position should be shutdown to keep balance. For cars and trains, the unbalance between the degradation states of the pair of wheels may not only accelerate the degradation process, but also result in serious consequences. With the development of electric vehicles, new balance problems in battery configuration and control are emerging. When the battery power difference between the left and right sides is too large, the electric vehicle may not be able to start.

This paper focuses on the maintenance optimization of a special balanced system- the train wheel. As one of the most important component of high-speed train, the state of the train wheel bears at least half of the responsibility for the derailment of the train ( $\mathrm{E}$ et al. (2018)). When the high-speed rail is running or turning, the flanges of the wheels and the rails are in contact and squeezed to generate force, which makes the wheels drive the train and turn. If the wheel flange thickness is too degraded, then the force generated by squeezing between the rail and flange may deform the flange; hence the wheel may deviate from the rail and fall off. If the wheel tread diameter is degraded under the safety threshold, then it may cause the wheel idling and the force between the pair unbalanced. Thus the wheels flange thickness and tread diameter have to keep in the safety range (Liang (2017), E et al. (2018)).

However due to the long mileages during the lifetime, the wheels flange and tread wear gradually. Then the thickness of flange and diameter of tread starts to reduce. Once the rail-wheel pair on the one side starts to degrade, the rail-wheel pair on the other side will be affected due to the unbalanced pressure and eventually starts to degrade. When the difference of tread diameter between the pair increases, the wheel with the larger diameter accelerates its degradation process. Rotary cut is a common technique that is applied in practice to restore the thickness of the flange of wheels in pair. The reprofiling action works due to the structure of the wheel and the tolerance of the reduction of the wheel tread. The schematic diagram is showed by Figure 1 (Liang (2017), E et al. (2018)).

Some researchers studied the maintenance policy on a single wheel. The time of applying preventive maintenance and the amount the thickness are the important maintenance decision variables to be optimized. Markov and semi-Markov decision process are used to decide the optimal state to be restored by reprofiling by dividing the amount of reprofiling into limited states (E et al. (2018), Costa et al. (2021)). Some others focus on the reliability evaluation of wheel state by data-driven methods (Chi et al. (2020)). 


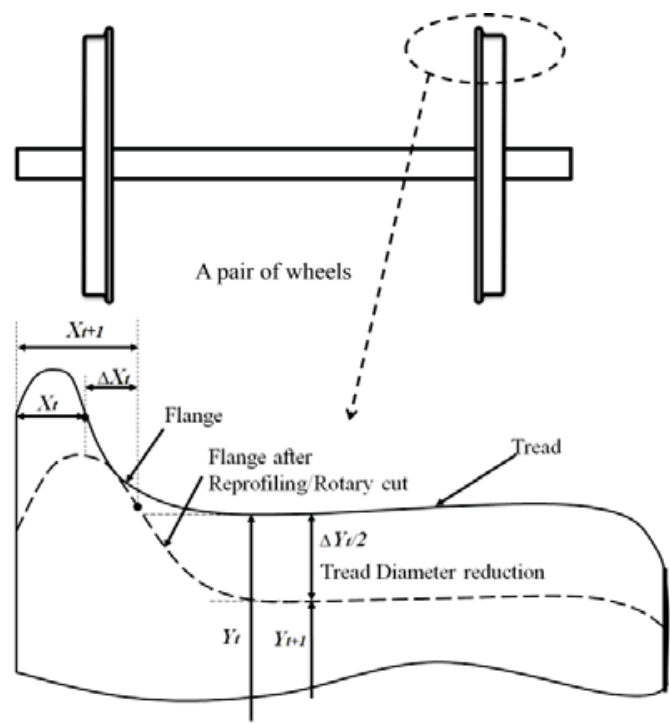

Fig.1. The schematic diagram of flange reprofiling by rotary cut of the tread of wheel

In this study, based on the industrial practice the pair of train wheels is considered as a subsystem. The dependency effect of the difference between the wheels diameter on the degradation is included. The Wiener process with timedependent and state-dependent drift and diffusion coefficients is used to model the degradation of the flange and the tread. A maintenance policy considering both the periodic maintenance and preventive maintenance threshold is proposed.

\section{Model Description}

Without considering maintenance action, the flange and tread of a wheel degrade individually. Due to the shape, structure and material, the degradation processes of flange and tread are random and modelling by a linear Wiener process with drift due to its statistical properties (Liang (2017)). Considering the dependence caused by the difference of wheel diameter of a pair of wheels, in this paper the degradation processes of flange $X_{t}$ and tread $Y_{t}$ are extended as follows:

$$
\begin{aligned}
& d X_{t}=\mu_{X}\left(X_{t}, t\right) d t+\sigma_{X}\left(X_{t}, t\right) d W_{t}^{X} \\
& d Y_{t}=\mu_{Y}\left(Y_{t}, t\right) d t+\sigma_{Y}\left(Y_{t}, t\right) d W_{t}^{Y}
\end{aligned}
$$

where $X_{t}$ is referred as an Ito process (diffusion process) and is subject to a normal distribution with mean $\mu_{X}\left(X_{t}, t\right)$ and variance $\sigma_{X}{ }^{2}\left(X_{t}, t\right)$ (the similar setting and assumption for $Y_{t}$ in Eq (2)). $W_{t}^{\bullet}$ is a standard Brownian motion which has the same form both in Eq (1) and Eq (2). The wheels of the same pair follow the distribution function with the same law of distribution. More property of Wiener process can be found in Dong(2020).

The maximum tolerance of thickness reduction of flange is referred as $X_{\alpha}$. The maximum tolerance of diameter reduction of wheel tread is referred as $Y_{\beta}$. The maximum tolerance of the difference of wheel diameter of pair is referred as $Y_{\gamma}$. The ratio of thickness restore of flange by reducing the diameter of wheel through rotary cut is referred as $\rho$.

\section{Failure Scenarios and Maintenance Policy}

During long-term running one of the following scenarios could happen to the pair of wheels: 1) the diameter reduction of at least one of the pair exceeds $Y_{\beta} ; 2$ ) difference of wheel diameter of a pair of wheels exceeds $Y_{\gamma}$; 3) the thickness reduction of at least one of the pair exceeds $X_{\alpha}$. Thus based on the 3 scenarios, the maintenance is proposed.

\subsection{Failure of Wheel}

When scenario 1) occurs, i.e., the diameter reduction of at least one wheel of the pair exceeds $Y_{\beta}$. The risk of derailment increases and the damage of flange cannot be restored by reprofiling. Thus the current state can be regarded as wheel failure and the whole pair of wheels should be replaced by a new wheel pair.

\subsection{Difference of Wheel Diameter of a Pair of Wheels}

When scenario 2) occurs, i.e., the difference of wheel diameter of a pair of wheels exceeds $Y_{\gamma}$, it is preferable to decrease the difference of wheel diameter by rotary cutting on the wheel with larger diameter. As a result, the thickness of flange of the wheel profiled will increase.

\subsection{Failure of Flange}

When scenario 3) occurs, i.e., the thickness reduction of at least one wheel of the pair exceeds $X_{\alpha}$, the probability of derailment alarmingly increases ( $E$ et al. (2018)) and this state is referred to failure of flange. The flange failure can be restored by reprofiling if the diameter of the wheel still allows for it. Regardless of the label of the wheels (i.e., which one of the pair of wheels), the restored amount of thickness can be calculated as follows:

$$
\Delta X_{t}=\left\{\begin{array}{cc}
X_{t}, & Y_{t}+\Delta Y_{t}<Y_{\beta} \\
\left(Y_{\beta}-Y_{t}\right) \cdot \rho, & Y_{t}+\Delta Y_{t}>Y_{\beta}
\end{array}\right.
$$


where $\Delta Y$ is the amount that the diameter should be reduced. Thus the restore amount of flange is in $\left[0, X_{t}\right]$.

When mentioning the label of each wheel, the following conditions by Eq (4-5) should be satisfied:

$$
\begin{array}{r}
\left|\left(Y_{t}^{(1)}+\Delta Y_{t}^{(1)}\right)-\left(Y_{t}^{(2)}+\Delta Y_{t}^{(2)}\right)\right| \leq \eta Y_{\gamma} \\
\max \left(Y_{t}^{(1)}+\Delta Y_{t}^{(1)}, Y_{t}^{(2)}+\Delta Y_{t}^{(2)}\right)<Y_{\beta}
\end{array}
$$

where the superscript of $Y_{t}^{(1)}$ indicates the first wheel of pair. Notice that Eq (4) restricts the difference of wheels' diameter. $\eta$ is a range value to reinforce the constraint of Eq (4). Eq (5) restricts the possibility of wheel failure.

\subsection{Maintenance Policy Structure}

Assume that the pair of wheel is inspected periodically and the real condition of the wheel can be available every $\tau$ time unit. The preventive maintenance with decision variable $\lambda \in(0,1)$ can be implemented during the opportunity of inspection. The procedure is as follows:

At time $t=k \tau$,

1) If $\max \left(Y_{t}^{(1)}, Y_{t}^{(2)}\right)>Y_{\beta}$, then the pair of wheel fails and should be replaced by a new pair of wheel.

2) If $\max \left(Y_{t}^{(1)}-Y_{t}^{(2)}, Y_{t}^{(2)}-Y_{t}^{(1)}\right)>Y_{\gamma}$, then a preventive maintenance, i.e., rotary cut, should be implemented according to Eq (4-6) on the wheel whose diameter is larger.

3) If $\max \left(X_{t}^{(1)}, X_{t}^{(2)}\right)>X_{\alpha}$, then a flange failure occurs and a corrective maintenance is needed.

4) If $\lambda X_{\alpha}<\max \left(X_{t}^{(1)}, X_{t}^{(2)}\right)<X_{\alpha}, \quad \lambda \in(0,1)$, a preventive maintenance is needed.

Either corrective maintenance or preventive maintenance is realized by reprofiling implemented according to Eq (3-5). Then after maintenance $\left(X_{t}^{(1)}, X_{t}^{(2)}, Y_{t}^{(1)}, Y_{t}^{(2)}\right)$ are updated as follows:

$$
\left\{\begin{array}{l}
X_{t}^{(i)}=X_{t}^{(i)}-\Delta X_{t}^{(i)} \\
Y_{t}^{(i)}=Y_{t}^{(i)}+\Delta Y_{t}^{(i)}
\end{array},\right.
$$

where $i=1,2$.

If no such profiling can be implemented, i.e., the remaining acceptable wheel diameter cannot compensate the reduction of flange thickness, the pair of wheel is regarded as wheel failure.

Notice that theoretically the probability of the occurrence of three scenarios at the same time corresponding to the three steps of the maintenance policy is non-zero, but through simulations and statistical results the previous procedure ensures that the three scenarios cannot occurs at the same time. But scenario 2) and 3) may occur at the same time, which is included in the proposed maintenance policy.

According to the previous procedure of maintenance, the decision variables should be optimized is $(\tau, \lambda)$, i.e, the inspection and preventive maintenance period, the preventive maintenance threshold of flange. As parameter $\eta$ controls both the reduction of the difference of wheel diameter and the restored amount of flange, its effects on the maintenance policy are nonlinear. In this study the effect of $\eta$ will be analyzed by some numerical experiments without optimization. When the maintenance activities are considered, the degradation trajectories of the flange and the wheel are shown by Figure 2 .
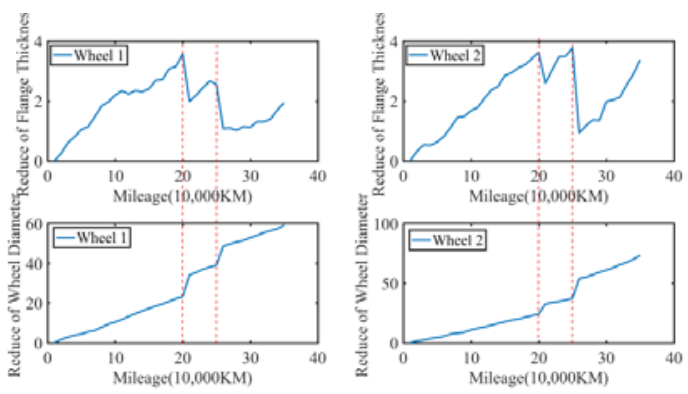

Fig.2. The degradation trajectories of the flange and the wheel with maintenance/reprofiling. The dash lines indicate the moment when reprofiling is implemented.

\subsection{Maintenance cost}

When the thickness reduction of at least one wheel exceeds the safety threshold of flange thickness, i.e., $\max \left(X_{t}^{(1)}, X_{t}^{(2)}\right)>X_{\alpha}$, or the diameter reduction of at least one wheel exceeds the safety threshold of wheel diameter, i.e., $\max \left(Y_{t}^{(1)}, Y_{t}^{(2)}\right)>Y_{\beta}$, the wheel pair is regarded as at the high risky running/function state and it is probably that the derailment would happen. In this paper, the cost of the so-called high risky running/function state is represented by the time duration being at this state multiplying the unit cost.

According to the renewal theorem, the average cost rate of maintenance of a pair of wheel is the cost of expected maintenance cycle over the length of expected cycle. In this paper the average cost rate is calculated according to Monte-Carlo simulations by counting the times of failures and preventive maintenances.

$E C(T)=\frac{E(C)}{E(T)}$ 


$$
E C(T) \approx \frac{N_{f}(T) C_{f}+D_{c}(T) C_{c}+N_{p}(T) C_{p}+N_{i}(t) C_{i}}{E(T)}
$$

where $N_{f}(T)$ is the number of wheel failure and meanwhile the number of replacement; $D_{c}(T)$ is the high risky running/functioning time of the wheel when either of wheel failure or flange failure occurs; $N_{p}$ is the number of preventive maintenance by increasing and thickness flange and reducing difference of wheel diameter; $N_{i}(t)$ is the number of condition inspection. $C_{f}, C_{p}$ and $C_{i}$ are unit cost of the previous counting numbers respectively. $C_{c}$ is the cost rate of high risky functioning $D_{c}(T)$.

Thus the maintenance decision problem is the following optimization problem:

$$
\left(\tau^{*}, \lambda^{*}\right)=\arg \min _{\tau, \lambda}(E C(T))
$$

\section{Simulations and Results analysis}

\subsection{Parameter Setting}

Referred to Liang (2017) and E et al. (2018), the parameters are set as follows: $X_{\alpha}=6, Y_{\beta}=70, Y_{\gamma}=10$, $\rho=0.4 . L_{X}=32$ and $L_{Y}=910$ are the initial thickness of flange and diameter of wheel tread respectively.

According to Eq (1-2), substituting the parameters the degradation process are as follows:

$$
\begin{aligned}
d X_{t}^{(1)}= & 0.15\left(1+\mathbf{I}_{\left\{Y_{t}^{(1)}>Y_{t}^{(2)}\right\}} c^{3}+X_{t}^{(1)} / L_{X}\right) d t \\
& +0.3\left[1+\left(X_{t}^{(1)} / L_{X}\right)^{2}\right] d W_{t}^{X} \\
d Y_{t}^{(1)}= & 1.2\left(1+\mathbf{I}_{\left\{Y_{t}^{(1)}>Y_{t}^{(2)}\right\}} c^{2}+Y_{t}^{(1)} / L_{Y}\right) d t \\
+ & 0.5\left[1+\left(Y_{t}^{(1)} / L_{Y}\right)^{2}\right] d W_{t}^{Y}
\end{aligned}
$$

where $c=\max \left(\left(Y_{t}^{(1)}-Y_{t}^{(2)}\right) / Y_{\gamma},\left(Y_{t}^{(2)}-Y_{t}^{(1)}\right) / Y_{\gamma}\right)$.

$W_{t}^{\bullet}$ is the standard Brownian motion which has the same form for $X_{t}$ and $Y_{t} \cdot X_{t}^{(2)}$ and $Y_{t}^{(2)}$ have the similar format as above.

\subsection{Results Analysis}

Notice that when a flange failure occurs, i.e, at least one of the flanges' thicknesses degrades to $X_{\alpha}=6$ and the high risky running cost $C_{c}=15$ per time unit should be included with the failure of flange. When the wheel fails, i.e., the diameter reduction of at least one wheels exceeds $Y_{\beta}=70$, or the flange thickness cannot be restored by reprofiling, the pair of wheels should be replaced with
$C_{f}=100$. The inspection cost and preventive maintenance cost are set as $C_{p}=20$ and $C_{i}=5$.

Table 1 . The average cost rate with different decision parameters $(\tau, \lambda)$ with the minimal average cost rate EC with $\eta=0.5, C_{f}=100, C_{c}$ =15, $C_{p}=20$ and $C_{i}=5$.

\begin{tabular}{c|cccccccccc}
$\lambda \tau$ & 5 & 10 & 15 & 20 & 25 & 30 & 35 & 40 & 45 & 50 \\
\hline 0.1 & 8.0 & 5.6 & 4.9 & 5.0 & 5.2 & 5.5 & 6.1 & 6.7 & 7.1 & 7.4 \\
\hline 0.2 & 7.5 & 5.5 & 4.9 & 5.0 & 5.2 & 5.5 & 6.1 & 6.7 & 7.1 & 7.4 \\
\hline 0.3 & 6.6 & 5.3 & 4.8 & 5.0 & 5.2 & 5.5 & 6.1 & 6.7 & 7.1 & 7.4 \\
\hline 0.4 & 6.0 & 5.0 & 4.8 & 5.0 & 5.2 & 5.5 & 6.1 & 6.7 & 7.1 & 7.4 \\
\hline 0.5 & 5.7 & 4.9 & 4.9 & 5.0 & 5.3 & 5.5 & 6.1 & 6.7 & 7.2 & 7.4 \\
\hline 0.6 & 5.4 & 4.9 & 5.1 & 5.2 & 5.4 & 5.6 & 6.1 & 6.7 & 7.2 & 7.4 \\
\hline 0.7 & 5.2 & 4.9 & 5.4 & 5.6 & 5.6 & 5.6 & 6.1 & 6.7 & 7.2 & 7.4 \\
\hline 0.8 & 5.2 & 5.2 & 5.7 & 6.0 & 6.0 & 5.8 & 6.1 & 6.7 & 7.1 & 7.4 \\
\hline 0.9 & 5.5 & 5.6 & 6.1 & 6.4 & 6.5 & 6.1 & 6.3 & 6.7 & 7.2 & 7.4 \\
\hline
\end{tabular}

According to Table.1, with the given $\eta=0.5$, the average long run cost rate changes with periodic inspection interval $\tau$ and preventive maintenance threshold parameter $\lambda$. The minimal cost rate achieves when $(\tau, \lambda)=(15,[0.3,0.4])$. When $C_{c}$ changes, the optimal inspection interval changes as well according to Fig. 3 and Fig.4.

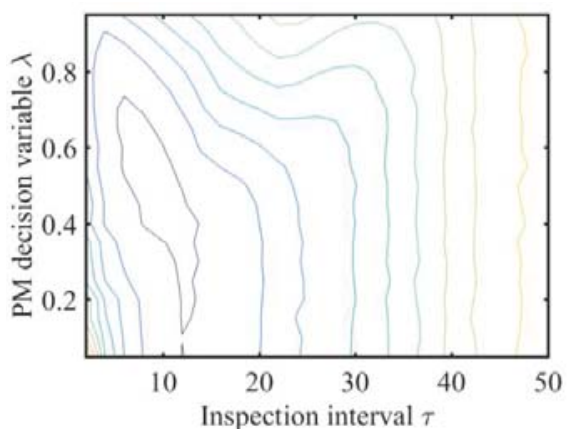

Fig.3. Contour of EC with pairs of $(\tau, \lambda)$ with $C_{c}=30$

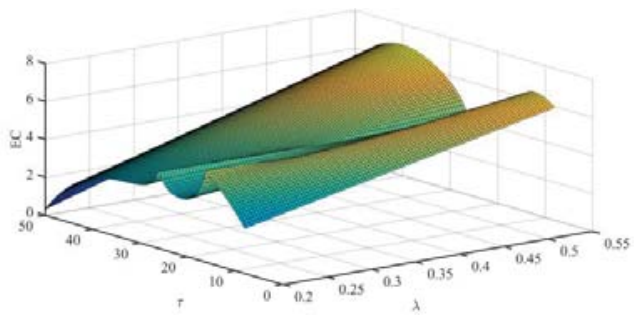

Fig.4. EC with $\left(\tau^{*}, \lambda *\right)$ when changing $C_{c}$ 
In order to observe the effect of the inspection/preventive interval on the average maintenance cost, Fig. 5 shows the change of EC with $\tau \in[1,50]$ and $C_{c}=15$ and 30 . The results confirm with the fact that when the inspection interval is too long, the failure may occur before the inspection/preventive maintenance.

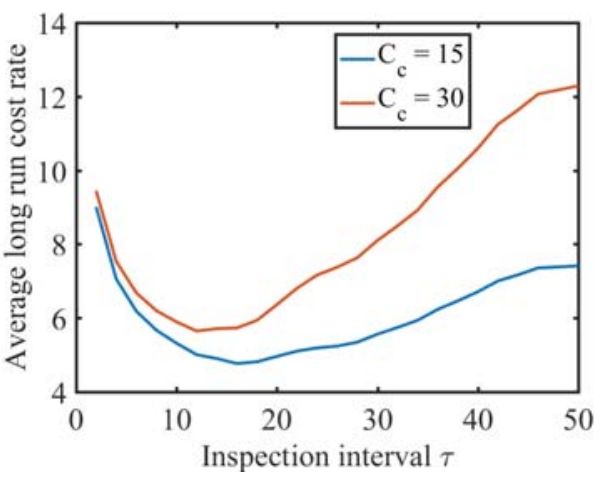

Fig. 5 The average long run cost rate with $\lambda=0.3$

\section{Conclusion}

Based on a pair of wheels of train as an example of balanced system with tolerance, this paper models the dependent degradation process by diffusion process and carries on some simulations to explore the performance of a preventive maintenance policy. The tolerance of the difference of wheel diameters is considered in this study and its effect on the maintenance policy is analyzed by simulations.

The preventive maintenance policy in this study is not very advanced, yet it makes an attentive analysis of the factors that affect on the reliability and maintenance cost of train wheel considering the industrial practice. Based on it, some more advanced maintenance policy which makes use of the residual life prediction on the level of the pair of wheels can be developed in future.

\section{Acknowledgement}

This work was supported by the National Natural Science Foundation of China Grant Nos. 71701163.

\section{References}

Gao, H., Cui, L. \& Yi, H. (2019). Availability analysis of k-outof-n: f repairable balanced systems with $m$ sectors. Reliability Engineering \& System Safety 191, 106572.

Zhao, X., Wu, C., Wang, X. \& Sun, J. (2020). Reliability analysis of k-out-of-n: $\mathrm{f}$ balanced systems with multiple functional sectors. Applied Mathematical Modelling 82, 108-124.

Fang, C. \& Cui, L. (2020). Balanced systems by considering multi-state competing risks under degradation processes. Reliability Engineering \& System Safety 205, 107252.

E, M., Li, B., Jiang, Z. \& Li, Q. (2018). An optimal reprofiling policy for high-speed train wheels subject to wear and external shocks using a semi-markov decision process. IEEE Transactions on Reliability 67(4), 1468-1481.

Liang, C. (2017). A maintenance model for high speed train wheel subject to internal degradation and external shocks. Prognostics \& System Health Management Conference (PHM-Harbin) 2017, 1-5.

Costa, M., Braga, J., Andrade, A. R. (2021). A data driven maintenance policy for railway wheelset based on survival analysis and Markov decision process. Quality and Reliability Engineering 37, 176-198.

Chi, Z., Chen, R., Huang, S., et al.(2020). Multi-State System Modeling and Reliability Assessment for Groups of Highspeed Train Wheels. Reliability Engineering \& System Safety 202,107026.

Dong, Q., Cui, L. \& Si, S. (2020). Reliability and availability analysis of stochastic degradation systems based on bivariate Wiener processes. Applied Mathematical Modelling 79, 414-433. 